\title{
Linearized model for optimization of coupled electricity and natural gas systems
}

\author{
Mathias SIRVENT ${ }^{1}$, Nikolaos KANELAKIS ${ }^{2}$, Björn GEIßLER ${ }^{1}$, \\ Pandelis BISKAS ${ }^{2}$ (i)
}

MPCE

\begin{abstract}
In this paper a combined optimization of a coupled electricity and gas system is presented. For the electricity network a unit commitment problem with optimization of energy and reserves under a power pool, considering all system operational and unit technical constraints is solved. The gas network subproblem is a medium-scale mixed-integer nonconvex and nonlinear programming problem. The coupling constraints between the two networks are nonlinear as well. The resulting mixed-integer nonlinear program is linearized with the extended incremental method and an outer approximation technique. The resulting model is evaluated using the Greek power and gas system comprising fourteen gas-fired units under four different approximation accuracy levels. The results indicate the efficiency of the proposed mixedinteger linear program model and the interplay between computational requirements and accuracy.
\end{abstract}

CrossCheck date: 9 January 2017

Received: 18 January 2016/Accepted: 9 January 2017/Published online: 25 April 2017

(C) The Author(s) 2017. This article is an open access publication

$\bowtie$ Pandelis BISKAS

pbiskas@auth.gr

Mathias SIRVENT

mathias.sirvent@fau.de

Nikolaos KANELAKIS

nkanelak@auth.gr

Björn GEIßLER

bjoern.geissler@math.uni-erlangen.de

1 Discrete Optimization, Friedrich-Alexander-Universität Erlangen-Nürnberg (FAU), Cauerstr. 11, 91058 Erlangen, Germany

2 School of Electrical and Computer Engineering, Aristotle University of Thessaloniki, 54124 Thessaloniki, Greece
Keywords Electricity system, Natural gas system, Mixedinteger (non) linear programming, Extended incremental method, Outer approximation

\section{Introduction}

The importance of electric gas units (mainly combined cycle gas turbines, CCGTs) has been increased during the recent years due to their attractive features as flexible resources in view of the increasing renewable energy sources (RES) penetration. This is attributed to their increased efficiency, small environmental footprint and high flexibility.

The popularity of CCGTs has given rise to common energy infrastructure considerations by regulators and system analysts, identifying the strong interdependence between the electricity and gas system in technical, economic and operational terms.

Despite their common nature as energy transmission systems, the operation of the natural gas system is extremely complex, employing a large-scale, highly nonconvex and nonlinear problem structure (comprising a group of nonlinear algebraic equations), which can be modeled as a nonconvex mixed-integer nonlinear program (MINLP) [1]. Compared to this, the electricity problem, which is a unit commitment problem with optimization of energy and reserves, can be modeled as a mixed-integrater program (MIP) [2].

A review of the developed nonlinear models for combined consideration of the electricity and gas systems is given in [3] and [4]. In all models, there is a nonlinear coupling constraint linking the electricity system with the gas system.

The majority of the research in the gas system focuses on simplifications of the nonlinear equations that govern 
the physics of gas flow as well as the general representation of the technical components (e.g. compressor model) of the gas transmission system. A current useful and efficient way to alleviate the burden of the nonconvex MINLP gas problem is the application of mixed integer linear programming techniques for piecewise linearization. In this way, the nonlinear formulation is approached by approximated linearized functions that render the problem as a MIP, which enables the computation of global optima within fast execution times. The most comprehensive article that studies the advantages and drawbacks of various MIP formulations for the gas optimization problem is [5], where different piecewise linearization approaches are tested for different test cases in both dynamic and stationary conditions in order to derive conclusions about the more accurate and faster approximations. Based on the results, the authors argue about the effectiveness of the incremental method and further apply it in [6] and [7] in order to linearize the transient flow equations for a combined electricity-gas model that is applied on the Belgian high-calorific gas network, where the effects of initial linepack in system operation are examined. A simpler linearization approach can be seen in [8] and [9] for a stochastic unit commitment and a combined optimal power flow model, respectively. Simple electricity-gas conversion factors are applied to all studies and the compressor machine modeling is also reduced to a compression factor. The incremental method is again applied in [10] for stationary gas flow within a large decomposition framework.

A literature overview of the linearized combined models discussed above is given in Table 1, according to the linearization approach applied, the modeling of the coupling constraint and the compressor, as well as the configuration of the gas flow. The main contributions introduced in this paper with respect to the current literature are the following.

1) The extended incremental method is used for the linearization of the gas physics [11]. This linearization method builds on the simple incremental method, exhibiting enhanced features and advantages as compared to standard piecewise linear approximation methods.

2) The nonlinear and nonconvex compressor operating range is linearized by an outer approximation approach. This approach often yields a rather detailed approximation (as opposed to simple linear approximations typically used in the literature), while keeping the computational overhead relatively low compared to the piecewise linearization of multivariate nonlinearities with the extended incremental method.

3) The coupling constraints (originally quadratic functions) are linearized with the extended incremental method as well, whereas in the literature a simple linear conversion ratio is applied.

Section 2 describes the nonconvex MINLP, whereas Sect. 3 presents the corresponding linearized model (MIP). In Sect. 4, several computations are presented and discussed afterwards. Lastly, in Sect. 5 the computational results are summurized.

\section{Problem statement}

The nonconvex MINLP is composed of an electricity (MIP) and a gas model (nonconvex MINLP) which are described in Sects. 2.1 and 2.2, respectively. The coupling of these models via nonlinear functions is explained in Sect. 2.3.

\subsection{Electricity problem}

In this paper, the day-ahead scheduling performed by the transmission system operator involves the solution of the unit commitment problem with simultaneous optimization of energy and reserves. The problem objective function concerns the minimization of the total as-bid cost for energy and for the provision of reserves plus the unit start-up/shut-down costs, subject to the following system operational constraints and unit technical constraints, for

Table 1 Literature overview for linearized models

\begin{tabular}{llll}
\hline Reference & Gas physics & $\begin{array}{l}\text { Compressor/ } \\
\text { coupling }\end{array}$ & Configuration \\
\hline Correa-Posada and Sánchez-Martín [6, 7] & Incremental & Single linear & Transient \\
Correa-Posada and Sánchez-Martín [8,9] & Piecewise & Single linear & Stationary \\
Wang et al. [10] & Incremental & Single linear & Stationary \\
Current & Extended & Linearized & Stationary \\
& Incremental & Range/extended & incremental \\
\hline
\end{tabular}


all dispatch periods $t \in T$ (typically, the dispatch period is $1 \mathrm{~h})[2]$.

1) Unit start-up/desynchronization constraints.

2) Unit minimum up/down time constraints.

3) Logical status of unit commitment constraints.

4) Unit power output normal and AGC limits.

5) Unit reserve capability constraints.

6) Unit ramp-up/down constraints.

7) The power balance equations.

8) System reserve requirements.

Due to the presence of binary variables for unit commitment and automatic generation control (so-called AGC) status, the electricity problem constitutes a MIP model. The objective value is defined as the electricity system cost $C_{\mathrm{e}}$ for all dispatch periods $t \in T$.

\subsection{Gas problem}

The optimization of gas transport networks has been recently described in [1] and in the survey article [12]. The main objective is to find cost optimal operations, which stick to the required pressure and flow bounds. As gas mainly flows from higher to lower pressures, it is required to install compressor machines in order to increase the gas pressure again. These compressions can be controlled by the transmission system operator, which adds discrete aspects to the problem among others. The physics of gas transport are driven by differential equations, which are simplified herein for the description of a steady-state problem. In this subsection, the gas model is analytically described. It should be noted that in all descriptions of Sect. 2.2 the dispatch period index is deliberately ignored.

The gas network is modeled via a directed graph $G=$ $(V, A)$ with node set $V$ and $\operatorname{arc}$ set $A$. The set of gas nodes $u, v \in V$ is partitioned into the set of entry nodes $V_{+}$(where gas is supplied), the set of exit nodes $V_{-}$(where gas is discharged) and the set of inner nodes $V_{0}$ (without any supply or discharge). Entries can be LNG (liquefied natural gas) entries $V_{\text {lng }}$ or standard pipeline entries $V_{\text {ent }}$. Exits can be electric gas loads (power plants) $V_{\mathrm{e}}$ or non-electric gas loads $V_{\text {ne }}$. The arcs are divided into pipes $A_{\text {pi }}$, control valves $A_{\mathrm{cv}}$ and compressor machines $A_{\mathrm{cm}}$.

For every arc $a=(u, v) \in A$, variables $q_{a}$ are defined, expressing the gas (mass) flow in arc direction when $q_{a}>0$ and vice versa, and having certain lower and upper bounds.

$q_{a} \in\left[q_{a}^{-}, q_{a}^{+}\right] \quad \forall a \in A$

Every node $u \in V$ has ingoing and outgoing arcs, defined as $\delta^{\text {in }}(u)$ and $\delta^{\text {out }}(u)$, respectively. For every node $u \in V$, a pressure variable $p_{u}$ and a flow variable $q_{u}$ is defined with certain upper and lower bounds.

$$
\begin{cases}p_{u} \in\left[p_{u}^{-}, p_{u}^{+}\right] & \forall u \in V \\ q_{u} \in\left[q_{u}^{-}, q_{u}^{+}\right] & \forall u \in V\end{cases}
$$

The flow variables $q_{u}$ represent the gas inputs (nonpositive) and outputs (nonnegative) at node $u$. Flow bounds are implicitly computed from pressure bounds and are useful only for preproccessing purposes. The nodal flow variables are fixed to zero for inner nodes.

$q_{u}^{-}=q_{u}^{+}=0 \quad \forall u \in V_{0}$

Moreover, there is a constraint for the outputs at nonelectric gas loads. The forecasted gas demand $d_{u}$ for every $u \in V_{\text {ne }}$ should be fulfilled as far as possible. If this is not possible, a so-called shedding variable $s_{u}$ measures this deviation according to the following:

$q_{u}+s_{u}=d_{u} \quad \forall u \in V_{\mathrm{ne}}$

Using the flow variables, the mass flow conservation equation is formulated as follows:

$\sum_{a \in \delta^{\text {in }}(u)} q_{a}-\sum_{a \in \delta^{\text {out }}(u)} q_{a}=q_{u} \quad \forall u \in V$

The pipes $A_{\mathrm{pi}}$ are used to transport gas through the network and are specified by their length $L_{a}$, diameter $D_{a}$, and integral roughness $k_{a}$. Here the differential equations of gas dynamics come into play. These gas dynamics within a single pipe are described by the Euler equations [13]. However, finishing well-known simplification steps, an analytic formula of pressure and flow in a pipe can be given via the Weymouth equation [1], Chapter 6.

$p_{v}^{2}-p_{u}^{2}=\frac{L_{a} \lambda_{a} R s z_{a} T}{A_{a}^{2} D_{a}}\left|q_{a}\right| q_{a} \quad \forall a \in A_{\mathrm{pi}}$

The specific gas constant $R s$ depends on the gas composition, while the friction factor $\lambda_{a}$ is calculated from the diameter and the integral roughness using the formula of Nikuradse [14], and $z_{a}$ is the average compressibility factor. Furthermore, the cross-sectional area of pipe $a \in A_{\text {pi }}$ can be calculated with the help of the diameter as follows:

$A_{a}=D_{a}^{2} \frac{\pi}{2} \quad \forall a \in A_{\mathrm{pi}}$

$z_{a}$ of pipe $a \in A_{\mathrm{pi}}$ is calculated with an average pressure on a pipe regarding the bounds, thus

$p_{m, a}=\frac{\max \left\{p_{u}^{-}, p_{v}^{-}\right\}+\min \left\{p_{u}^{+}, p_{v}^{+}\right\}}{2} \quad \forall a \in A_{\mathrm{pi}}$

The formula of the American Gas Association is used to calculate the compressibility factor [15]: 
$z(p, T)=1+\alpha p$

where $\alpha$ constitutes a parameter given as a function of the pseudocritical pressure $p_{c}$ and the pseudocritical temperature $T_{c}$ :

$\alpha=0.257 \frac{1}{p_{c}}-0.533 \frac{T_{c}}{p_{c} T}$

The isothermal case assumes constant temperature $T$, which is used for that model. Now, (8)-(10) are combined for the calculation of $z_{a} \cdot z_{a}=z\left(p_{m, a}, T\right), \forall a \in A_{\mathrm{pi}}$.

Moreover, so-called short pipes are defined as auxiliary network elements. They are used to model multiple entries at a single entry node or to model multiple exits at a single exit node and can be interpreted as pipes $a=(u, v) \in A_{\mathrm{pi}}$ with length $L_{a}=0$ and therefore from (6), $p_{u}=p_{v}$ can be concluded.

Control valves $A_{\mathrm{cv}}$ are used to decrease gas pressure. These elements are modeled with some discrete aspects, since they can be operated in different modes (active, bypass and closed). A closed control valve decouples the in- and outflow pressures and prohibits any gas flow through $a=(u, v) \in A_{\mathrm{cv}}$. The bypass mode ensures the flow through the control valve, but does not influence pressure, while the active mode ensures the desired pressure decrease within a controllable range $\Delta_{a} \in\left[\Delta_{a}^{-}, \Delta_{a}^{+}\right]$:

$\begin{cases}a \text { is active } & \Rightarrow p_{v}=p_{u}-\Delta_{a} \\ a \text { is in bypass mode } & \Rightarrow p_{v}=p_{u} \\ a \text { is closed } & \Rightarrow q_{a}=0\end{cases}$

Compressor machines $A_{\mathrm{cm}}$ are necessary to increase pressure, in order to transport gas over long distances. As control valves, a compressor machine can be either active, closed or in bypass mode. The working ranges of turbo compressors are typically described by characteristic diagrams, as shown for example in Fig. 1, where the admissible operating range is depicted in green.

The horizontal axis in the diagram is labeled with the volumetric flow rate at inlet conditions.

$Q_{a}=\rho_{u} q_{a}$

where $\rho_{u}$ denotes the density at node $u$. Density is related to pressure via the equation of state:

$p_{u}=\rho_{u} R s T_{u} z_{u}$

In the isothermal model presented in this paper, the gas temperature at the inlet $T_{u}$ is constant. The vertical axis is labeled with the specific change in adiabatic enthalpy

$H_{a}^{\mathrm{ad}}=R s T_{u} z_{u} \frac{\kappa}{\kappa-1}\left[\left(\frac{p_{v}}{p_{u}}\right)^{\frac{\kappa-1}{\kappa}}-1\right]$

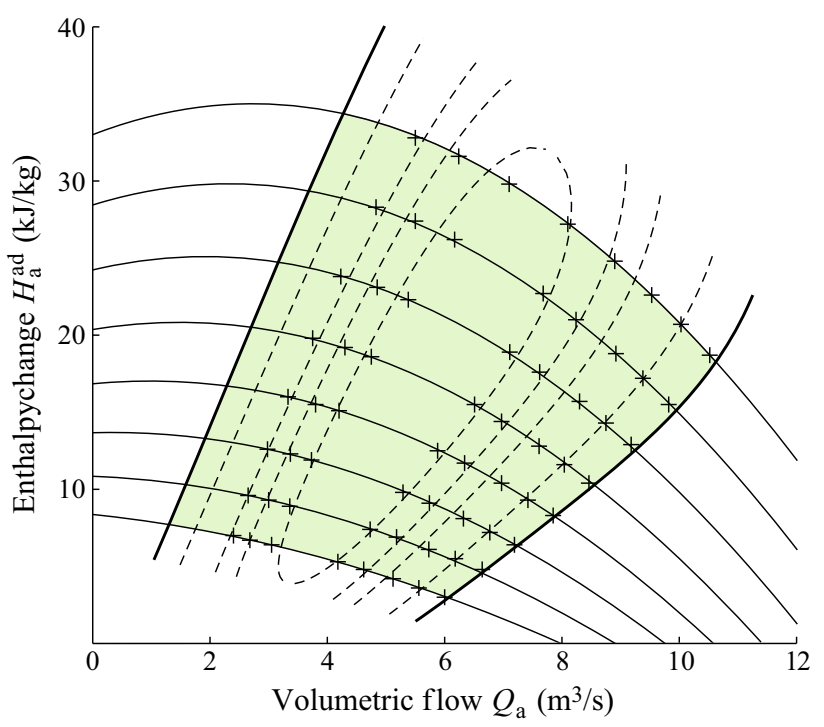

Fig. 1 Characteristic diagram of turbo compressor

The isentropic exponent $\kappa=1.38$ is also a constant in the model.

In Fig. 1, the dashed lines represent isolines for the adiabatic efficiency of the compressor and the thin solid lines are isolines for compressor speed. The upper and lower bounding curve of the feasible region is given by the isolines for minimum and maximum speed. To the left, the working range is bounded by the surgeline. The right boundary curve is called chokeline. Both surgeline and chokeline are depicted with thick solid lines. All these curves result from (bi-)quadratic least squares fits of the measured data points, which are depicted as crosses in Fig. 1. The four bounding can be written as nonlinear inequalities of the form:

$f_{a}^{i}\left(Q_{a}, H_{a}^{\mathrm{ad}}\right) \leq 0 \quad \forall a=(u, v) \in A_{\mathrm{cm}}, \quad i=1,2,3,4$

The power required for compression is given by

$P_{a}=\frac{q_{a} H_{a}^{\mathrm{ad}}}{\eta_{a}} \quad \forall a=(u, v) \in A_{\mathrm{cm}}$

where, $\eta_{a}$ denotes the adiabatic efficiency of the compressor, which is considered constant in this paper. Further details on the modeling of compressors can be found in [1], Sect. 2.

Since the objective is to minimize costs, the combination of the compressor costs and the shedding costs are calculated. It should be noted that the gas supply cost of the power plants is included in their variable cost, which is implicitly incorporated within their energy offer prices in the electricity model; thus, they are not included again in the objective function of the gas optimization problem. The 
compressor costs depend on the power of compressor machine $P_{a}$, while the shedding costs depend on $s_{u}$. The corresponding parameters $c_{a}$ for $P_{a}$ and $\gamma$ for $s_{u}$ represent the cost coefficient for compressor machine $a \in A_{\mathrm{cm}}$ and the cost term (penalty price) for shedded gas flow, respectively, and are used to compute the objective value. The overall optimization problem of the gas system is formulated as follows:

$\min \sum_{a \in A_{\mathrm{cm}}} c_{a} P_{a}+\sum_{u \in V_{\mathrm{ne}}} \gamma s_{u}$

s.t. pressure and flow bounds $(1)-(3)$

shedding at non-electric gas loads (4)

mass flow conservation (5)

pressure decrease in pipe (6)

control valve model (11)

compressor model (12)-(16)

The overall compressor costs $C_{\mathrm{c}}$ and the overall costs due to shedded gas flows $C_{\mathrm{s}}$ for all dispatch periods $t \in T$ can now be formulated with $C_{\mathrm{c}}=\sum_{t \in T} \sum_{a \in A_{\mathrm{cm}}} c_{a} P_{a}^{t}$ and $C_{\mathrm{s}}=\sum_{t \in T} \sum_{u \in V_{\mathrm{ne}}} \gamma s_{u}^{t}$.

Thus (17)-(23) can be extended to all dispatch periods:

$$
\min C_{\mathrm{c}}+C_{\mathrm{s}}
$$

s.t. $\quad$ gas constraints $(18)-(23)$ for all $t \in T$

Problem (24)-(25) yields a nonconvex MINLP, since the control valves and the compressor machines include discrete variables, and (6) and (12)-(16) include nonlinear functions as equations and nonconvex inequalities. Note that Problem (24)-(25) is block diagonal, where each block is made up of the variables and constraints of a single discrete time step.

\subsection{Coupling of gas and electricity problem}

The two energy systems are coupled through the gas consumption of the electric gas loads. The coupling constraint can be mathematically formulated with

$u_{u}^{t} \frac{a_{u}+b_{u} e_{u}^{t}+c_{u}\left(e_{u}^{t}\right)^{2}}{H}=q_{u}^{t} \quad \forall u \in V_{\mathrm{e}}, \forall t \in T$

The binary variable $u_{u}^{t}$ and continuous variable $e_{u}^{t}$ express the commitment status of electric gas load $u \in V_{\mathrm{e}}$ and the electricity production of unit $u \in V_{\mathrm{e}}$, at dispatch period $t \in T$, respectively. The fuel consumption coefficients of electric gas loads $u \in V_{\mathrm{e}}$, which are $a_{u}, b_{u}$, and $c_{u}$, as well as the heating value of natural gas $H$, are needed to calculate the conversion. For simplicity, it is assumed that the composition of the supplied gas does not vary among the system entries.
Intertemporal constraints between dispatch periods are only present in the electricity unit commitment problem in Sect. 2.1, while the gas model (24)-(25) can be separated for every dispatch period. The overall optimization problem is expressed as a minimization problem where the respective costs of the two systems are combined to express the overall objective function. It is a nonconvex MINLP:

$\min C_{\mathrm{e}}+C_{\mathrm{c}}+C_{\mathrm{s}}$

s.t. electricity constraints in chapter 2.1

gas constraints (25)

coupling constraints (26)

\section{Linearized formulation}

Two linearization techniques are used to tackle the nonlinearities of (27)-(30). The nonlinear functions in (6), (16), and (26) are linearized with the so-called extended incremental method [11]. The nonlinear and nonconvex set of (15) is linearized with outer approximation constraints [16].

Both techniques ensure the relaxation property, which means that each solution to the nonlinear model (27)-(30) is also feasible for the linearized model. This is important as pure approximations tend to be infeasible even if the underlying nonlinear model is feasible [17].

\subsection{Extended incremental method}

First of all, it should be mentioned that the incremental method as basis of the extended incremental method is just one of various methods to linearize nonlinear functions. MIP-relaxations may be formulated with alternative methods, for instance with a convex combination model that introduces only a number of extra binary variables and constraints that is logarithmic in the number of breakpoints [18]. However, the incremental method is used in this paper, since it performs best for gas transport problems $[5,17,19]$.

The extended incremental method works by first introducing a new finitely bounded variable for each nonlinear term, and by then computing a piecewise linear approximation of it. The approximation is constructed such that an a priori given upper bound on the approximation is satisfied, while introducing as less breakpoints as possible. Using the known values of the resulting approximations error, a MIP-relaxation model for the nonlinearities is derived.

To be more precise, for a constraint of the form $\boldsymbol{c}^{\mathrm{T}} \boldsymbol{x}+f(y)=0$, with $f: \mathbf{R} \rightarrow \mathbf{R}$ nonlinear, and finitely bounded $y \in\left[y_{\min }, y_{\max }\right]$, a new variable $z$ is introduced, 
reformulating the constraint as $\boldsymbol{c}^{\mathrm{T}} \boldsymbol{x}+z=0$, and a piecewise linear approximation $\Phi(y)=z$ of $f(y)$ over $\left[y_{\min }, y_{\max }\right]$ with $\max _{y_{\min } \leq y \leq y_{\max }}|\Phi(y)-f(y)| \leq \varepsilon$ is computed.

Assuming that $\Phi(y)$ is defined over $n$ intervals, subdivided by breakpoints $y_{\min }=b_{0}<b_{1}<\ldots<b_{n-1}=y_{\max }$, the overall MIP-relaxation model of the constraint $\boldsymbol{c}^{\mathrm{T}} \boldsymbol{x}+$ $f(y)=0$ is reformulated as follows:

$\left\{\begin{array}{l}y=b_{0}+\sum_{i=1}^{n}\left(b_{i}-b_{i-1}\right) \delta_{i} \\ z=f\left(b_{0}\right)+\sum_{i=1}^{n}\left(f\left(b_{i}\right)-f\left(b_{i-1}\right)\right) \delta_{i}+e \\ \delta_{i+1} \leq w_{i} \leq \delta_{i} \quad i=1,2, \ldots, n-1 \\ \delta_{i} \in[0,1] \\ w_{i} \in\{0,1\} \\ e \in[-\varepsilon, \varepsilon] \\ z \in \mathbf{R} .\end{array}\right.$

Each of the $\delta$-variables is associated with a discretization interval. With the so-called filling condition $\delta_{i+1} \leq w_{i} \leq \delta_{i}$, we make sure that $\delta_{i}>0$ requires $\delta_{j}=1$ for all $j<i$ and that $\delta_{i}<1$ requires $\delta_{j}=0$ for all $j>i$. This way, there can be at most one index $i$ of an interval with $0<\delta_{i}<1$. In this case, $y$ lies within the $i^{\text {th }}$ interval. Assuming $e=0$, the point $(y, z)$ would lie on the $i^{\text {th }}$ line segement of the piecewise linear function. However, with $e \in[-\varepsilon, \varepsilon]$, the point $(y, z)$ lies within a box of height $2 \varepsilon$ that encloses the graph of the piecewise linear function. Since $\varepsilon$ has been chosen such that also the graph of the approximated nonlinear function is contained in the boxes, model (31) yields a relaxation.

The extended incremental method is used for the nonlinear terms in (6), (16), and (26). In order to rewrite (16) as a sum of univariate nonlinear expressions, we plug in Eq. (14) and apply the standard reformulation $x y=-((x-$ $\left.y)^{2}-x^{2}-y^{2}\right) / 2$ for bilinear expression two times, before the extended incremental method is applied. It should be also noted that that after linearization, (26) is still a product of a binary variable $u_{u}^{t}$ and a newly defined continuous variable according to (31). This resulting nonlinear term can be linearized without an error by the well-known bigM method.

\subsection{Outer approximation}

Outer approximation is a well-known linearization method for convex nonlinear programs [16]. In this paper, it is used for the linearization of (15), although a nonconvex operating range is involved. The idea is to compute a convex envelope of the operating range and to add tangential hyperplanes to the envelope during optimization. More details on this technique can be found in [1].

\section{Case study}

The developed model is tested on the Greek power system together with the Greek natural gas transmission system. The description of these networks is given in Sect. 4.1, while the computational results are presented in Sect. 4.2. First, the linearized formulation of Sect. 3 is tested in Sect. 4.2.1. Afterwards, the results are compared with a single electricity model in Sect. 4.2.2, where the gas network is not taken into account.

\subsection{Real-world test system}

The Greek power system is operated by ADMIE (Independent Power Transmission Operator S.A.) [20]. The generation mix and the marginal cost range for each unit category is presented on Table 2. In the test case, each thermal unit is considered to submit one price/ quantity offer at its minimum variable operating cost. The opportunity cost of hydro units (cost of replacement of a thermal $\mathrm{MWh}$ ) is considered for the generation of the respective energy offers. This has been considered slightly higher than the variable cost of the most expensive thermal units. Indeed, in countries with a low portfolio of hydro resources (e.g., in Greece), such opportunity cost is significantly high, since hydro units essentially operate as peakers (peak-shaving units), replacing expensive (high-cost) thermal units. Finally, the system load is considered inelastic.

The Greek natural gas transport network is operated by the Greek natural gas transmission operator DESFA [21]. Figure 2 shows the network topology and Table 3 gives some statistical information about the network.

The system constitutes a radial network consisting of three entry points, namely two pipeline entries in the northern part of the country in Sidirokastro and Kipi, and

Table 2 Greek power system generating units' data with unit type, number of unit types, installed capacity, and marginal cost range

\begin{tabular}{lcll}
\hline Unit type & Number & $\begin{array}{l}\text { Capacity } \\
(\mathrm{MW})\end{array}$ & $\begin{array}{l}\text { Marginal cost } \\
(€ / \mathrm{MWh})\end{array}$ \\
\hline Lignite units & 16 & 4302 & $43.18-51.20$ \\
CCGTs & 11 & 5026 & $76.30-85.00$ \\
OCGTs & 3 & 147 & 150 \\
Hydro units & 15 & 2997 & 90 \\
RES & 50 & 4191 & - \\
\hline
\end{tabular}


an LNG entry in the southern part, in the island of Revythousa close to the metropolitan area of Athens. The electric gas loads are mainly located in the south, while the system is supplemented by a compressor station in Nea Messimvria (close to Thessaloniki) to maintain gas flows from the northern entries. Moreover, a control valve is located at the Lavrio branch.

\subsection{Computational results}

All computations have been performed on a computer with an Intel i7-4770 CPU with 4 cores running at $3.4 \mathrm{GHz}$ each, and 16 GB of main memory.

The electricity model was developed with GAMS 24.4.6 [22], while the linearized gas model was built with the $\mathrm{C}++$ software Framework LaMaTTo ++ [23]. GAMS was used to combine these models and to linearize the coupling. As MIP solver, Gurobi 6.0.4 was used [24]. A MIP gap tolerance of $0.01 \%$ was selected. Moreover, a time limit of $1 \mathrm{~h}$ was set as the electricity unit commitment problem has to be solved in $1 \mathrm{~h}$.

As instances, the days with highest non-electric gas loads $\sum_{t \in T} \sum_{u \in V_{\text {ne }}} d_{u}^{t}$ out of all days of every quarter of the years 2013-2015 were chosen yielding in total twelve instances.

Regarding the approximation accuracy, two varying parameters have been allowed. The first parameter $\varepsilon_{1}$ is the maximum error of the approximation of the nonlinear

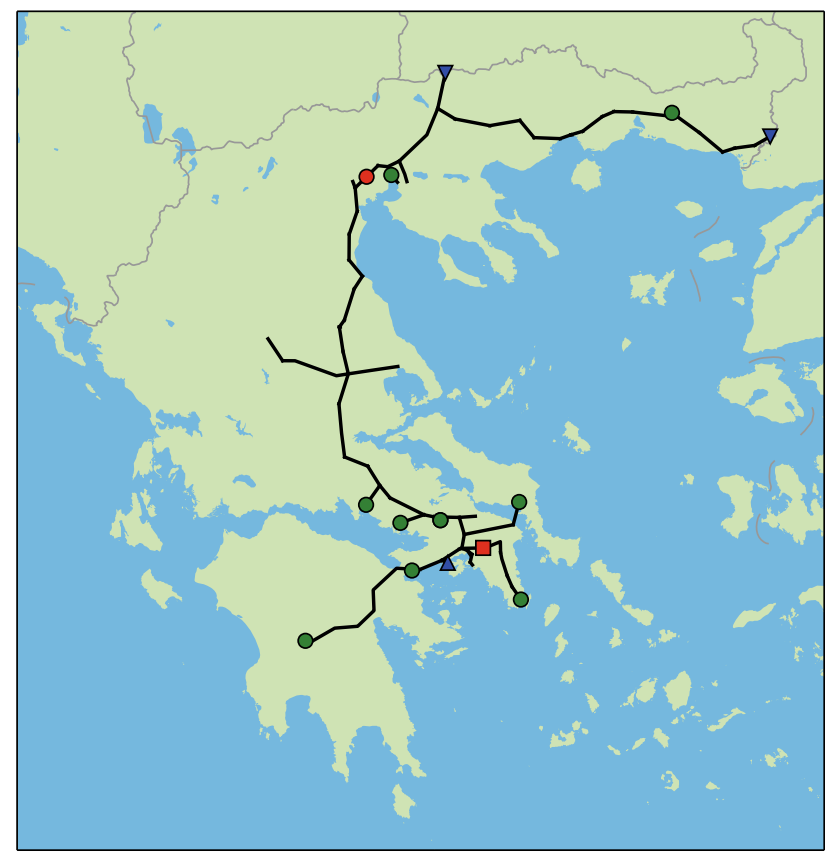

$\checkmark$ Pipeline entries; $\triangle$ LNG entry; • Compressor machine

- Control valve; $\bullet$ Electric gas loads
Table 3 Data of Greek gas network with 134 nodes and 133 arcs

\begin{tabular}{lcllc}
\hline 134 nodes & & & 133 arcs & \\
\cline { 1 - 2 } \cline { 5 - 5 } Type & Quantity & & Type & Quantity \\
\hline Pipeline entries & 2 & & Pipes & 86 \\
LNG entries & 1 & & Short pipes & 45 \\
Non-electric & 34 & & Control valves & 1 \\
Gas loads & & & & \\
Electric gas & 11 & & Compressor & 1 \\
loads & & & machines & \\
Inner nodes & 86 & & &
\end{tabular}

pressure loss function (6) in bar. The second parameter $\varepsilon_{2}$ is the maximum error allowed for $e_{u}^{2}$ in $\mathrm{MW}^{2}$. Four different pairs of values for $\varepsilon_{1}$ and $\varepsilon_{2}$ are compared in the computations that are performed as shown in Table 4.

Moreover, preprocessing according to chapter 6 in [1] is used for the gas problem. These methods tighten the bounds of flow variables and reduce the number of linearized functions. If a squared variable $x^{2}$ occurs in the problem without the use of the variable $x$ itself, then $x^{2}$ can be used without a linearization and $x$ can be calculated a posteriori.

Regarding the entries, two different scenarios were examined.

Case I For the first case, the historical gas entry distribution of Kipi, Sidirokastro and Revythousa from 2013 to 2015 was averaged. Therefore, Kipi and Revythousa provide upto $20 \%$ of the gas each and the remaining upto $60 \%$ are supplied at Sidirokastro. In the present model, the optimizer is allowed to deviate from this distribution by at most ten percent at each entry.

Case II Another case with closed LNG entry is implemented, due to its periodic maintenance or outage. The entry distribution of Kipi and Sidirokastro can be chosen freely by the optimizer in this case. However, it is required that at least $20 \%$ of consumed gas has to be supplied from Kipi.

\subsubsection{Linearized formulation}

The average solution time and problem size for each accuracy level from Table 4 is given in Table 5, while the

Table 4 Approximation accuracy levels

\begin{tabular}{lrr}
\hline Label & \multicolumn{1}{c}{$\varepsilon_{1}$} & \multicolumn{1}{c}{$\varepsilon_{2}$} \\
\hline A & 1.5 & 1 \\
B & 2.5 & 10 \\
C & 5.0 & 100 \\
D & 15.0 & 1000 \\
\hline
\end{tabular}

Fig. 2 Greek gas network 
Table 5 Average number of continuous variables, discrete variables, constraints, and average solution time. The numbers in parentheses denote the number of instances that hit the time limit

\begin{tabular}{|c|c|c|c|c|c|c|c|c|}
\hline \multirow[t]{2}{*}{ Accuracy } & \multicolumn{4}{|l|}{ Case I } & \multicolumn{4}{|l|}{ Case II } \\
\hline & $\begin{array}{l}\text { Continuous } \\
\text { variables }\end{array}$ & Discrete & Constraints & $\begin{array}{l}\text { Solution } \\
\text { time (s) }\end{array}$ & $\begin{array}{l}\text { Continuous } \\
\text { variables }\end{array}$ & Discrete & Constraints & $\begin{array}{l}\text { Solution } \\
\text { time (s) }\end{array}$ \\
\hline A & 136570.00 & 54913.58 & 186217.00 & $500.18(0)$ & 134467.50 & 53826.33 & 183706.50 & 1762.09 (5) \\
\hline B & 81756.17 & 27506.67 & 108507.17 & $96.05(0)$ & 80845.67 & 27015.42 & 107188.67 & $917.54(2)$ \\
\hline $\mathrm{C}$ & 61030.83 & 17144.00 & 80413.83 & $26.57(0)$ & 60696.33 & 16940.75 & 79671.33 & $516.91(1)$ \\
\hline D & 54284.83 & 13771.00 & 71411.83 & $8.72(0)$ & 54212.83 & 13699.00 & 70931.83 & $142.51(0)$ \\
\hline
\end{tabular}

Table 6 Electricity costs $C_{\mathrm{e}}$, compressor costs $C_{\mathrm{c}}$, shedding costs $C_{\mathrm{s}}$, and solution time for instance 18/02/2015

\begin{tabular}{|c|c|c|c|c|c|c|c|c|}
\hline \multirow[t]{2}{*}{ Accuracy } & \multicolumn{4}{|l|}{ Case I } & \multicolumn{4}{|l|}{ Case II } \\
\hline & $C_{\mathrm{e}}(€)$ & $C_{\mathrm{c}}(€)$ & $C_{\mathrm{s}}(€)$ & Solution time (s) & $C_{\mathrm{e}}(€)$ & $C_{\mathrm{c}}(€)$ & $C_{\mathrm{s}}(€)$ & Solution time (s) \\
\hline A & 4155106.61 & 1918.72 & 0.00 & 593.54 & 4652407.31 & 4563.49 & 900463.23 & 3600.00 \\
\hline B & 4155105.52 & 1587.34 & 0.00 & 166.86 & 4642512.18 & 4408.67 & 900418.07 & 3600.00 \\
\hline $\mathrm{C}$ & 4155095.11 & 1219.04 & 0.00 & 34.65 & 4642326.27 & 3856.23 & 899957.85 & 3600.00 \\
\hline D & 4155000.91 & 865.70 & 0.00 & 10.05 & 4642212.69 & 2912.82 & 895779.05 & 1089.00 \\
\hline
\end{tabular}

day with the highest non-electric gas load, the 18/02/2015, is presented in Table 6 in detail.

Table 5 shows the growing amount of variables and constraints for tighter linearizations leading to higher solution times. While the number of variables and constraints only slightly deviates from the average within each row, the solution time is in general higher for higher nonelectric gas loads. Moreover, Case II turns out to be harder than Case I, which can be seen from the average solution time and the higher number of instances that hit the time limit.

Table 6 shows an instance, where solution times are higher than the averages from Table 5. The overall objective function (sum of $C_{\mathrm{e}}, C_{\mathrm{c}}$, and $C_{\mathrm{s}}$ ) is always higher for tighter approximations due to reduced feasible sets. This is mainly driven by the compressor costs $C_{\mathrm{c}}$ for Case I and by the compressor costs $C_{\mathrm{c}}$ and especially the shedding costs $C_{\mathrm{s}}$ for Case II. This can be verified by the average power of the compressor in every dispatch period for all instances, as shown in Fig. 3 for Case I and II. The average power is in general higher for tighter approximations and moreover higher for higher non-electric gas loads, thus especially during the evening. Higher compressor power leads to higher compressor costs due to (17)-(23).

As Case II prohibits incoming flow from the south, all exits, e.g. the electric gas loads in the south, have to be served by the northern entries. This leads to higher compressor costs for Case II compared to Case I, which follows from the necessary compression for the gas from the north. This can be seen in Fig. 3 again, where the average compressor power is much higher for Case II than Case I. Even if the compressor in Case II is able to fulfill the requirements of the electricity problem in Sect. 2.1, it is not able to guarantee the demands of the non-electric gas loads any more, thus $s_{u}>0$ in (4). Figure 4 visualizes the average of all these sheddings according to Case II. As all sheddings occur in the south, therefore a zoom is performed into this region in Fig. 4. Yellow circles represent non-electric gas loads without shedding, while magenta circles represent non-electric gas loads with shedding. The higher the averaged shedding, the bigger the radius of these circles. As the LNG entry is closed for Case II, the non-electric gas loads in the south will suffer according to the model.

\subsubsection{Comparison with single electricity model}

An additional way to depict the effect of the combined optimization of the two systems is by comparing it with the single electricity model, which is the solution of the electricity problem in Sect. 2.1 without any consideration of the coupling to the gas network. The solution of the single electricity model constitutes the optimal values of the energy production of all power plants, including CCGTs as electric gas loads. The differences of these values compared to the values of the combined optimization problem 


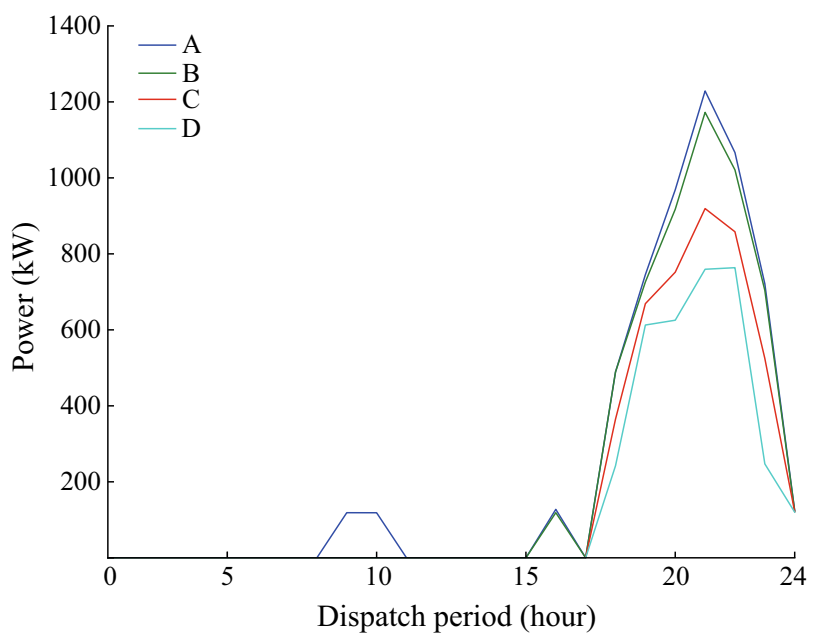

(a) Case I

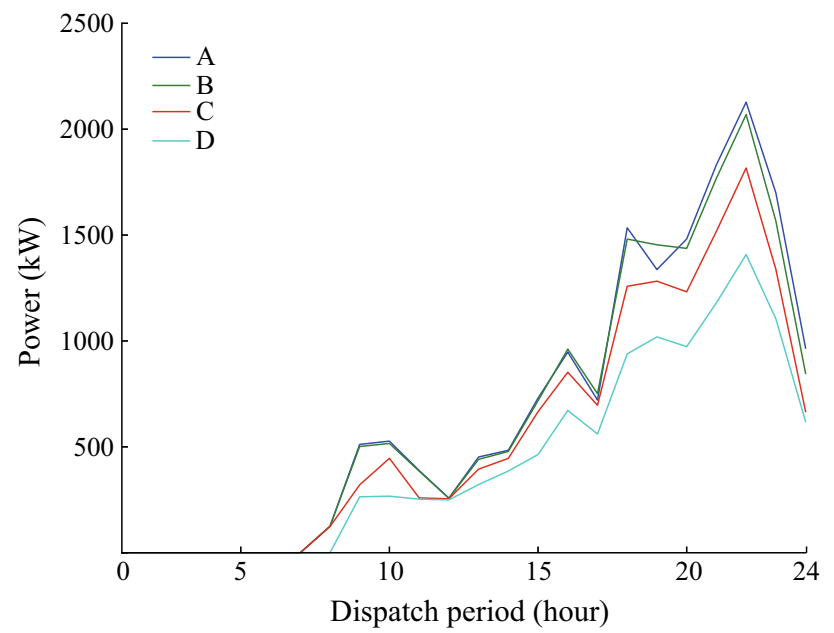

(b) Case II

Fig. 3 Average power of compressor for every dispatch period and accuracy level

are investigated. Figure 5 shows the average difference of all values per hour for all instances and accuracy levels.

It can be seen that the average production of the electric gas loads is reduced for many dispatch periods for both cases. The sharpest decline takes place during the evening peak hours and is even more evident for Case II.

The reduced energy production can be attributed to the fact that the gas network cannot allocate the desired amount of gas to the electric gas loads according to the optimal solution of the single electricity model. The difference is more evident in Case II when the supply capacity of the gas network is further reduced.

The lost production of the gas-fired power plants is compensated by an increase from the other, more expensive electricity production sources. This leads to higher electricity costs $C_{\mathrm{e}}$, which can be seen in Table 7 for both cases.

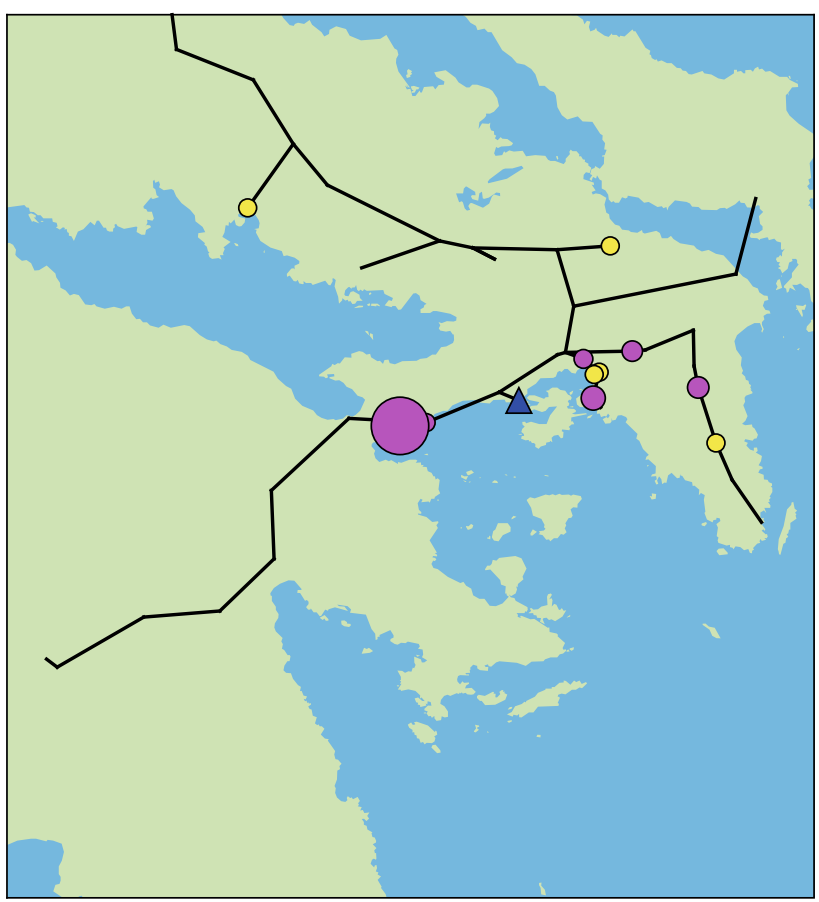

$\triangle$ LNG entry; Non-electric gas loads with shedding O Non-electric gas loads without shedding

Fig. 4 Southern part of the network from Fig. 2 without the control valve

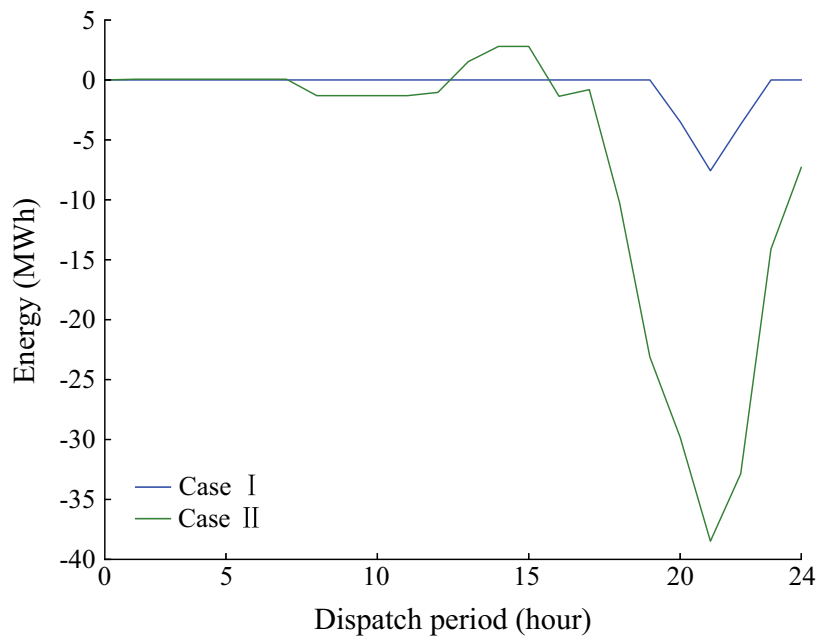

Fig. 5 Average difference of produced power by each CCGT between single electricity model and MIP for every dispatch period

Table 7 Average increase of $C_{\mathrm{e}}$ for both cases

\begin{tabular}{lll}
\hline Accuracy & Case I $(€)$ & Case II $(€)$ \\
\hline A & 2536.80 & 118771.41 \\
B & 2534.28 & 117864.75 \\
C & 2530.43 & 117841.05 \\
D & 2520.40 & 117779.13 \\
\hline
\end{tabular}




\section{Conclusion}

In this paper, the coupled optimization of electricity and natural gas systems was investigated for the real-world test system of Greece. The extended incremental method, as well as the outer approximation method, were applied for the linearization of the nonlinear equations that govern the physics of gas transport and for the coupling constraint of the two energy systems. Four different discretization accuracy levels were selected and the experiments were conducted on real system data for two distinct test cases. The computed results showed the effectiveness of the proposed combined optimization method by depicting.

1) The increase of objective function costs and solution times with respect to tighter approximations for all instances examined.

2) The appearance of shedded non-electric gas loads during certain instances, caused by the need to provide the demand of the electric gas loads, in order to maintain the electricity system balance.

3) The effect of the gas system's constraints on the electricity model, which was expressed in the increase of the electricity unit commitment cost.

This work is intended to act as a basis for further investigation into computations and comparisons of the coupled optimization problem, with additional consideration of stochasticity for the electricity system and the implementation of instationary gas flows for the gas system.

Acknowledgements This research has been performed as part of the Energie Campus Nürnberg. We also acknowledge funding through the DFG SFB/Transregio 154, Subprojects A05 and Z01.

Open Access This article is distributed under the terms of the Creative Commons Attribution 4.0 International License (http:// creativecommons.org/licenses/by/4.0/), which permits unrestricted use, distribution, and reproduction in any medium, provided you give appropriate credit to the original author(s) and the source, provide a link to the Creative Commons license, and indicate if changes were made.

\section{References}

[1] Koch T, Hiller B, Pfetsch ME, Schewe L (eds) (2015) Evaluating gas network capacities. SIAM-MOS series on Optimization. SIAM, pp xv +351

[2] Simoglou CK, Biskas PN, Bakirtzis AG et al (2010) Optimal self-scheduling of a thermal producer in short-term electricity markets by MILP. IEEE Trans Power Syst 25(4):1965-1977

[3] Biskas PN, Kanelakis NG, Papamatthaiou A et al (2016) Coupled optimization of electricity and natural gas systems using augmented Lagrangian and an alternating minimization method. Int J Electr Power Energy Syst 80:202-218
[4] Rubio R, Ojeda-Esteybar D, Ano O et al (2008) Integrated natural gas and electricity market: a survey of the state of the art in operation planning and market issues. In: Proceedings of the 2008 IEEE/PES transmission and distribution conference and exposition, Latin America, 13-15 Aug 2008, pp 1-8

[5] Correa-Posada CM, Sánchez-Martín P (2014) Gas network optimization: a comparison of piecewise linear models, Technological Research Institute (IIT) ICAI School of Engineering. Comillas Pontifical University, Madrid

[6] Correa-Posada CM, Sánchez-Martín P (2015) Integrated power and natural gas model for energy adequacy in short-term operation. IEEE Trans Power Syst 30(6):3347-3355

[7] Correa-Posada CM, Sánchez-Martín P (2015) Security-constrained unit commitment with dynamic gas constraints. In: 2015 IEEE power energy society general meeting, Denver, USA, 26-30 Jul 2015, 5 pp

[8] Correa-Posada CM, Sánchez-Martín P (2013) Stochastic contingency analysis for the unit commitment with natural gas constraints. In: Proceedings of powertech (POWERTECH), Grenoble, France, 16-20 Jun 2013, 6 pp

[9] Correa-Posada CM, Sánchez-Martín P (2015) Integrated power and natural gas model for energy adequacy in short-term operation. IEEE Trans Power Syst 29(4):1780-1787

[10] Wang C, Wei J, Wang J et al (2016) Robust defense strategy for gas-electric systems against malicious attacks. https://arxiv.org/ abs/1603.00832. Accessed 15 Jan 2016

[11] Geißler B, Martin A, Morsi A et al (2012) Using piecewise linear functions for solving MINLPs. In: Proceedings of mixed integer nonlinear programming, the IMA volumes in mathematics and its applications, Springer, New York 154:287-314

[12] Ríos-Mercado RZ, Borraz-Sánchez C (2015) Optimization problems in natural gas transportation systems: a state-of-the-art review. Appl Energy 147:536-555

[13] Feistauer M (1993) Mathematical methods in fluid dynamics. Pitman monographs and surveys in pure and applied mathematics 67. Longman Scientific \& Technical, New York

[14] Nikuradse J (1993) Strömungsgesetze in rauhen Rohren. Forschungsheft auf dem Gebiete des Ingenieurwesens. VDIVerlag, New York

[15] Králik J, Stiegler P, Vostrý Z et al (1988) Dynamic modeling of large-scale networks with application to gas distribution, vol 6 . Studies in automation and control. Elsevier, Amsterdam

[16] Duran MA, Grossmann IE (1986) An outer-approximation algorithm for a class of mixed-integer nonlinear programs. Math Program 36:307-339

[17] Geißler B (2011) Towards globally optimal solutions for MINLPs by discretization techniques with applications in gas network optimization. Ph.D, Friedrich Alexander Universität, Erlangen Nürnberg (FAU)

[18] Nemhauser G, Vielma J (2011) Modeling disjunctive constraints with a logarithmic number of binary variables and constraints. Math Program 128:49-72

[19] Morsi A (2013) Solving MINLPs on loosely-coupled networks with applications in water and gas network optimization. Ph.D, Friedrich Alexander Universität, Erlangen Nürnberg (FAU)

[20] ADMHE http://www.admie.gr Accessed 15 June 2016

[21] DESFA http://www.desfa.gr Accessed 15 June 2016

[22] GAMS development corporation general algebraic modeling system (GAMS) (2013) Release 24.4.6, Washington, DC, USA, http://www.gams.com/ Accessed 15 June 2016

[23] LaMaTTO++ (2015) A framework for modeling and solving mixed-integer nonlinear programming problems on networks. http://www.mso.math.fau.de/edom/projects/lamatto.html Accessed 15 June 2016

[24] Gu Z, Rothberg E, Bixby R (2015) Gurobi optimizer reference manual, Version 6.0.4. Gurobi Optimization Inc, Houston 
Mathias SIRVENT received his Master of Science Degree from the Department of Mathematics, Friedrich-Alexander-Universität Erlangen-Nürnberg, Germany, in 2013. Currently, he is a Ph.D. student with the same university at the Chair of Economics, Discrete Optimization, and Mathematics. His research interests are mixedinteger nonlinear programming especially gas network optimization.

Nikolaos KANELAKIS received his Dipl. Eng. Degree from the Department of Electrical and Computer Engineering, Aristotle University, Thessaloniki, in 2012. Currently, he is a Ph.D. student with the same university. His research interests are power and natural gas system operation and optimization theory applications in power systems.

Björn GEIßLER received his Dipl. Inform. Degree from the Department of Computer Science, TU Darmstadt, Germany, in
2007. In 2011, he received his Ph.D. from the Department of Mathematics, Friedrich-Alexander-Universität Erlangen-Nürnberg, Germany. Currently, he is working as postdoc researcher at the Chair of Economics, Discrete Optimization, and Mathematics again at Friedrich-Alexander-Universität Erlangen-Nürnberg.

Pandelis BISKAS received the Dipl.-Eng. and Ph.D. degrees from the Department of Electrical and Computer Engineering, Aristotle University of Thessaloniki, Greece, in 1999 and 2003, respectively. From March 2005 till July 2009 he was a wholesale market expert at the Hellenic TSO, Market Operation Department. Currently, he is an Assistant Professor at the Aristotle University of Thessaloniki, in the Department of Electrical and Computer Engineering. His research interests are in power system operation and control, electricity market operational and regulatory issues, and electricity market simulation and modeling. 